\title{
Long non-coding RNA SNHG1 relieves microglia activation by downregulating miR-329-3p expression in an in vitro model of cerebral infarction
}

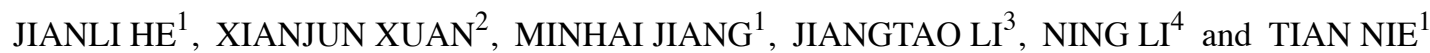 \\ ${ }^{1}$ Department of Neurology, Affiliated Hangzhou First People's Hospital, Zhejiang University School of Medicine, \\ Hangzhou, Zhejiang 310006; ${ }^{2}$ The Fourth Clinical College, Zhejiang Chinese Medical University, Hangzhou, \\ Zhejiang 310000; Departments of ${ }^{3}$ General Surgery and ${ }^{4}$ Urology, Affiliated Hangzhou First People's Hospital, \\ Zhejiang University School of Medicine, Hangzhou, Zhejiang 310006, P.R. China
}

Received February 4, 2021; Accepted July 7, 2021

DOI: $10.3892 /$ etm.2021.10581

\begin{abstract}
Following cerebral infarction, activated microglia cells can release a large amount of inflammatory cytokines, thereby exacerbating neuronal damage. It has been demonstrated that the long non-coding RNA small nucleolar RNA host gene 1 (SNHG1) exerts a protective effect against cerebral infarction. However, its specific role in cerebral infarction and underlying mechanism have yet to be fully elucidated. The present study aimed to investigate the effects of the SNHG1 and microRNA (miR)-329-3p in cerebral infarction and to determine the underlying molecular mechanisms. An in vitro oxygen-glucose deprivation (OGD) model was established using the BV-2 microglial cell line. The mRNA expression levels of SNHG1 and miR-329-3p were analyzed using reverse transcription-quantitative PCR and the protein expression levels of cleaved caspase- 3 and caspase- 3 were detected using western blotting. The binding relationship between SNHG1 and miR-329-3p was predicted using starBase and verified using a dual luciferase reporter assay. The release of TNF- $\alpha$ and nitric oxide, as well as caspase-3 activity, were detected using appropriate commercial kits. Flow cytometry analysis was performed to measure cell apoptosis. The results of the present study revealed that the expression levels of SNHG1 were upregulated in the OGD-induced BV-2 cell model. miR-329-3p was discovered to directly target SNHG1, and its mRNA expression levels were downregulated in the OGD-induced BV-2 cell model. The SNHG1-plasmid downregulated miR-329-3p expression levels, while this effect
\end{abstract}

Correspondence to: Dr Tian Nie, Department of Neurology, Affiliated Hangzhou First People's Hospital, Zhejiang University School of Medicine, 261 Huansha Road, Hangzhou, Zhejiang 310006, P.R. China

E-mail: nietian1058@163.com

Key words: cerebral infarction, microglia, microRNA-329-3p, long non-coding RNA small nucleolar RNA host gene 1 was reversed by transfection with the miR-329-3p mimic. The overexpression of SNHG1 or knockdown of miR-329-3p inhibited OGD-induced BV-2 cell activation. In conclusion, the results of the present study suggested that SNHG1 may reduce microglial cell activity by regulating the expression of miR-329-3p, indicating its potential protective role in cerebral infarction.

\section{Introduction}

Cerebral infarction, also known as ischemic stroke, refers to the local ischemic necrosis or encephalomalacia of brain tissue caused by disruption to the blood supply, leading to ischemia and hypoxia (1-3). Cerebral infarction is the most common type of cerebrovascular disease, accounting for $\sim 70 \%$ of all cerebrovascular diseases (4). The most common clinical causes of cerebral infarction include the formation of a cerebral thrombus, lacunar infarction and cerebral embolism $(5,6)$. Although the different types may share common etiological characteristics, there also exhibit specific differences. Diseases and conditions commonly associated with cerebral infarction include diabetes, obesity, hypertension, rheumatic heart disease, arrhythmia, dehydration due to various reasons, several types of arteritis, shock and a rapid or excessive drop in blood pressure (7-9). Cerebral infarction is considered as a medical emergency and is associated with high disability and mortality rates. Therefore, it is crucial to devise more effective treatments for cerebral infarction. Previous studies have reported that microglia activation may promote the excessive release of numerous inflammatory cytokines, thereby exacerbating neuronal damage after an ischemic stroke $(10,11)$. Therefore, the present study conducted a series of experiments using microglia to further study their role in cerebral infarction and elucidate the underlying mechanism.

Long non-coding RNAs (lncRNAs) are a class of non-coding RNAs of $>200$ nucleotides in length, which are transcribed in most eukaryotic genomes $(12,13)$. To date, most lncRNAs have been classified; however, their precise functions require further investigations. An increasing number of studies have shown that lncRNAs play key roles in regulating 
growth and development, cell differentiation, subcellular structure distribution and evolutionary selection, in addition to their roles in numerous types of human disease, including vascular, neurological and inflammatory diseases, as well as cancer (14-16). In fact, numerous IncRNAs, such as metastasis-associated lung adenocarcinoma transcript 1 , maternally expressed 3, CDKN2B antisense RNA 1 and small nucleolar RNA host gene 1 (SNHG1), have been reported to be involved in regulating the pathogenesis of cerebral infarction (17-20). SNHG1 is a lncRNA, comprising 3,927 nucleotides, which is transcribed from the SNHG1 gene on chromosome 11 (21). Chen et al (20) found that SNHG1 exerted a protective effect against cerebral infarction by regulating the PI3K/AKT signaling pathway. Other studies have revealed that regulating the endogenous activation of microglia may represent a new target for ischemic stroke treatment $(22,23)$. In addition, an oxygen-glucose deprivation (OGD) model of BV-2 cells has been widely used to study cerebral infarction in vitro (24-26). However, to the best of our knowledge, whether SNHG1 participates in cerebral infarction by affecting the activation of microglia has not been investigated to date.

The present study was undertaken to investigate the effects of SNHG1 on the activation of microglia and further determine its underlying molecular mechanism. The results may provide novel insight into potential clinical strategies for the treatment of cerebral infarction.

\section{Materials and methods}

Cell culture and OGD model establishment. The BV-2 microglial cell line was purchased from the American Type Culture Collection. Cells were cultured in DMEM (HyClone; Cytiva) supplemented with $10 \%$ FBS (Gibco; Thermo Fisher Scientific, Inc.), and maintained at $37^{\circ} \mathrm{C}$ with $5 \% \mathrm{CO}_{2}$.

The establishment of the OGD model of BV-2 cells was performed according to a previous study (25). Briefly, BV-2 cells were cultured in serum/glucose-free DMEM with $95 \% \mathrm{~N}_{2}$ and $5 \% \mathrm{CO}_{2}$ at $37^{\circ} \mathrm{C}$ for 12,24 or $48 \mathrm{~h}$. Following incubation, the cells were collected to determine the levels of SNHG1 and miR-329-3p.

Reverse transcription-quantitative PCR (RT-qPCR). Total RNA was extracted from cells using TRIzol ${ }^{\circledR}$ reagent (Invitrogen; Thermo Fisher Scientific, Inc.). Total RNA was reverse transcribed into cDNA using a SuperScript ${ }^{\circledR}$ VILO $^{\mathrm{TM}}$ cDNA Synthesis kit (Invitrogen; Thermo Fisher Scientific, Inc.). qPCR was subsequently performed on a Prism 7000 Real-Time PCR Detection system (Applied Biosystems; Thermo Fisher Scientific, Inc.) using SYBR qPCR Master mix (Thermo Fisher Scientific, Inc.), according to the manufacturer's protocol. The primers used for the qPCR were purchased from Genscript and the primer sequences are listed as follows:

GAPDH, forward 5'-CTTTGGTATCGTGGAAGGACT C-3' and reverse, 5'-GTAGAGGCAGGGATGATGTTCT-3'; U6, forward, 5'-GCTTCGGCAGCACATATACTAAAAT-3' and reverse, 5'-CGCTTCACGAATTTGCGTGTCAT-3'; IncRNA SNHG1, forward, 5'-CCAAACTCAGGCACTGTATAG AT-3' and reverse, 5'-ACAGACACGAAGTGGAGTTATG-3'; and miR-329-3p, forward, 5'-GTGGAACAGACCTGGTAA AC-3' and reverse, 5'-CAAGTGCGAGTCGTGCAGT-3'. The following thermocycling conditions were used for the qPCR: Initial denaturation for $5 \mathrm{~min}$ at $95^{\circ} \mathrm{C}$, followed by 40 cycles of $95^{\circ} \mathrm{C}$ for $10 \mathrm{sec}$ and $60^{\circ} \mathrm{C}$ for $30 \mathrm{sec}$. The relative mRNA expression levels of SNHG1 and microRNA (miRNA/miR)-329-3p were calculated using the $2^{-\Delta \Delta \mathrm{Cq}}$ method (27), and GAPDH or U6 were used as the internal controls for normalization of SNHG1 and miR-329-3p expression, respectively.

Cell transfection. The SNHG1 sequence was synthesized based on the SNHG1 sequence and then sub-cloned into the pcDNA3.1 vector (SNHG1-plasmid; Shanghai GeneChem Co., Ltd.). The empty pcDNA3.1 vector was used as a control (control-plasmid). BV-2 cells were plated into 6-well plates for $24 \mathrm{~h}$, then transfected with $1 \mu \mathrm{g}$ control-plasmid, $1 \mu \mathrm{g}$ SNHG1-plasmid, $50 \mathrm{nM}$ mimic control (5'-UUCUCC GAACGUGUCACGUTT-3'; GeneCopoeia, Inc.), $50 \mathrm{nM}$ miR-329-3p mimic (3'UUUUUCCAAUCGACCCACACA A5'; GeneCopoeia, Inc.), $1 \mu \mathrm{g}$ SNHG1-plasmid + $50 \mathrm{nM}$ mimic control, $1 \mu \mathrm{g}$ SNHG1-plasmid + $50 \mathrm{nM}$ miR-329-3p mimic, $100 \mathrm{nM}$ inhibitor control (5'CAGUACUUUUGUGUAGUA CAA3'; GeneCopoeia, Inc.) or 100 nM miR-329-3p inhibitor (5'AAAAAGGUUAGCUGGGUGUGUU3'; GeneCopoeia, Inc.) using Lipofectamine ${ }^{\circledR} 2000$ reagent (Invitrogen; Thermo Fisher Scientific, Inc.). Following transfection for $24 \mathrm{~h}$, the cells were collected to determine the transfection efficiency using RT-qPCR.

To investigate the role of SNHG1 in OGD induced BV-2 cells, BV-2 cells were cultured in serum/glucosefree DMEM with $95 \% \mathrm{~N}_{2}$ and $5 \% \mathrm{CO}_{2}$ at $37^{\circ} \mathrm{C}$ for $48 \mathrm{~h}$, then the cells were transfected with control-plasmid, SNHG1-plasmid, SNHG1-plasmid + mimic control, or SNHG1-plasmid + miR-329-3p mimic at $37^{\circ} \mathrm{C}$ for another $24 \mathrm{~h}$. Cells were divided into the following six groups: i) Control group; ii) OGD group; iii) OGD + controlplasmid group; iv) OGD + SNHG1-plasmid group; v) OGD + SNHG1-plasmid + mimic control group; and vi) OGD + SNHG1-plasmid + miR-329-3p mimic group.

To investigate the role of miR-329-3p downregulation in OGD induced BV-2 cells, BV-2 cells were cultured in serum/glucose-free DMEM with $95 \% \mathrm{~N}_{2}$ and $5 \% \mathrm{CO}_{2}$ at $37^{\circ} \mathrm{C}$ for $48 \mathrm{~h}$, then the cells were transfected with inhibitor control or miR-329-3p inhibitor at $37^{\circ} \mathrm{C}$ for $24 \mathrm{~h}$. Cells were divided into the following four groups: i) Control group; ii) OGD group; iii) OGD + inhibitor group; and iv) OGD + miR-329-3p inhibitor group.

miRNA target analysis and dual luciferase reporter assay. The binding relationship between miR-329-3p and SNHG1 was identified using starBase (http://starbase.sysu.edu.cn/). The 3'-untranslated region (UTR) sequences of SNHG1 containing the target sequence of miR-329-3p were obtained by RT-qPCR and cloned into a pmirGLO vector (Promega Corporation) to construct the SNHG1-wild-type (WT) reporter gene vector. A SNHG1-mutated type (MUT) reporter gene vector was also constructed. The BV-2 cells were cultured for $24 \mathrm{~h}$, then co-transfected with the SNHG1-WT or SNHG1-MUT reporter gene vector and miR-329-3p mimic (3'-UUUUUCCAAUCGACCCACACAA-5') or mimic control (5'-UUCUCCGAACGUGUCACGUTT-3') using Lipofectamine 2000 reagent at $37^{\circ} \mathrm{C}$ for $48 \mathrm{~h}$. The relative 
A
SNHG1-WT
5'GUUUUUCAGAGAU - GGUGUGUC3'
miR-329-3p
|| | |||||||
SNHG1-MUT
3'UUUUUCCAAUCGACCCACACAA5'
5'GUUUUUCAGUCAA - CCACACAC3'
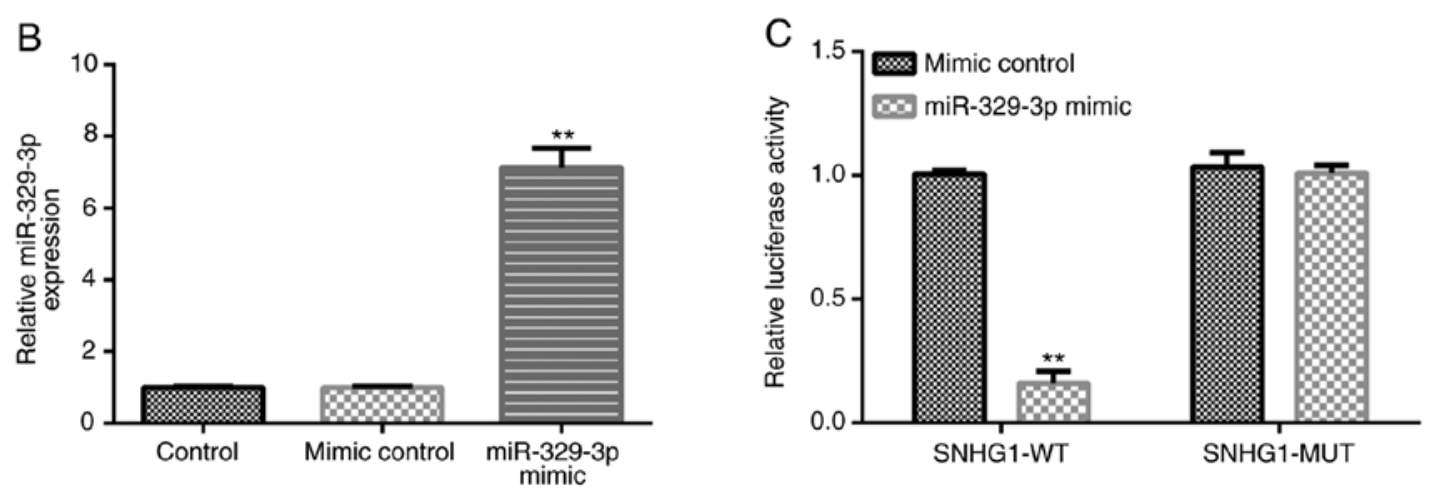

Figure 1. miR-329-3p directly targets SNHG1. (A) Predicted miR-329-3p binding sites in the 3'-UTR of SNHG1. (B) BV-2 cells were transfected with miR-329-3p mimic or mimic control for $48 \mathrm{~h}$, then the expression levels of miR-329-3p were analyzed using reverse transcription-quantitative PCR. (C) Dual luciferase reporter assay was used to verify the miR-329-3p binding sites in the 3'-UTR of SNHG1. ${ }^{* *} \mathrm{P}<0.01$ vs. mimic control. miR, microRNA; SNHG1, small nucleolar RNA host gene 1; UTR, untranslated region; WT, wild-type; MUT, mutant.

luciferase activity was measured using a Dual Luciferase Reporter assay system (Promega Corporation) according to the manufacturer's protocol. Luciferase activity was normalized to Renilla luciferase activity.

ELISA. Following $24 \mathrm{~h}$ of transfection, the cell culture supernatants were collected through centrifugation at $500 \mathrm{x}$ g at $4^{\circ} \mathrm{C}$ for $5 \mathrm{~min}$. Then, the levels of TNF- $\alpha$ in the supernatant of BV-2 cells were detected using an ELISA kit (cat. no. SMTA00B; $\mathrm{R} \& \mathrm{D}$ Systems, Inc.) according to the manufacturer's protocol.

Measurement of nitric oxide (NO) production. The production of $\mathrm{NO}$ was evaluated by detecting the nitrate levels in the cell supernatant. Briefly, equal volumes of BV-2 cell supernatant and Griess reagent were mixed and incubated for $10 \mathrm{~min}$ at room temperature. The absorbance was measured at a wavelength of $550 \mathrm{~nm}$ using a microplate reader (Bio-Rad Laboratories, Inc.).

Flow cytometric analysis of apoptosis. Flow cytometry wasused to detect the levels of cell apoptosis using Annexin V-FITC/PI apoptosis detection kit (Beyotime Institute of Biotechnology). Briefly, the transfected cells were collected by trypsinization after transfection and resuspended in $1 \mathrm{X}$ binding buffer. Then, $100 \mu \mathrm{l}$ cell suspension was incubated with $5 \mu \mathrm{l}$ Annexin V-FITC and $5 \mu 1$ PI (Beyotime Institute of Biotechnology) according to the manufacturer's protocol. Apoptotic cells were analyzed using a FACSCalibur flow cytometer (BD Biosciences) and FlowJo software (version 7.2.4; FlowJo LLC).

Detection of caspase-3 activity. Caspase-3 activity was measured using a colorimetric assay kit (Beyotime Institute of Biotechnology) according to the manufacturer's protocol.

Western blotting. Following incubation for $24 \mathrm{~h}$, total protein was extracted from BV-2 cells using RIPA lysis buffer (Beyotime Institute of Biotechnology) and centrifugation at $10,000 \mathrm{xg}$ for $15 \mathrm{~min}$ at $4^{\circ} \mathrm{C}$. Total protein was quantified using a BCA protein assay kit (Bio-Rad Laboratories, Inc.) and separated via 10\% SDS-PAGE. The separated proteins were subsequently transferred onto a PVDF membrane and blocked with PBS-0.1\% Tween-20 (PBST) containing 5\% non-fat milk at room temperature for $1 \mathrm{~h}$. The membranes were then incubated with the following primary antibodies at $4^{\circ} \mathrm{C}$ overnight: Anti-cleaved caspase-3 (cat. no. 9664; dilution 1:1,000; Cell Signaling Technology, Inc.), anti-caspase-3 (cat. no. 14220; dilution 1:1,000; Cell Signaling Technology, Inc.) or anti-GAPDH (cat. no. 5174; dilution 1:1,000; Cell Signaling Technology, Inc.). Following primary antibody incubation, the membrane was washed with PBST three times and incubated with the secondary antibody [goat anti-rabbit IgG H\&L (HRP) preadsorbed; cat. no. 97080; dilution 1:5,000; Abcam] for $1 \mathrm{~h}$ at room temperature. Protein bands were visualized using an ECL substrate (Cytiva) on an Amersham ImageQuant UV western blotting system (Cytiva) according to the manufacturer's instructions.

Statistical analysis. Statistical analysis was performed using the SPSS software (version 18.0; SPSS, Inc.). Data are presented as the mean \pm SD of three independent experiments. Statistical differences between groups were determined using an unpaired Student's t-test or one-way ANOVA followed by Tukey's post hoc test. $\mathrm{P}<0.05$ was considered to indicate a statistically significant difference.

\section{Results}

SNHGl is a direct target gene of miR-329-3p. Bioinformatics analysis using starBase identified a binding site between SNHG1 and miR-329-3p (Fig. 1A). Compared with the mimic control group, transfection with the miR-329-3p mimic significantly upregulated miR-329-3p expression levels in BV-2 cells (Fig. 1B). Then, the binding site between SNHG1 and miR-329-3p was confirmed using a dual luciferase reporter assay (Fig. 1C). 

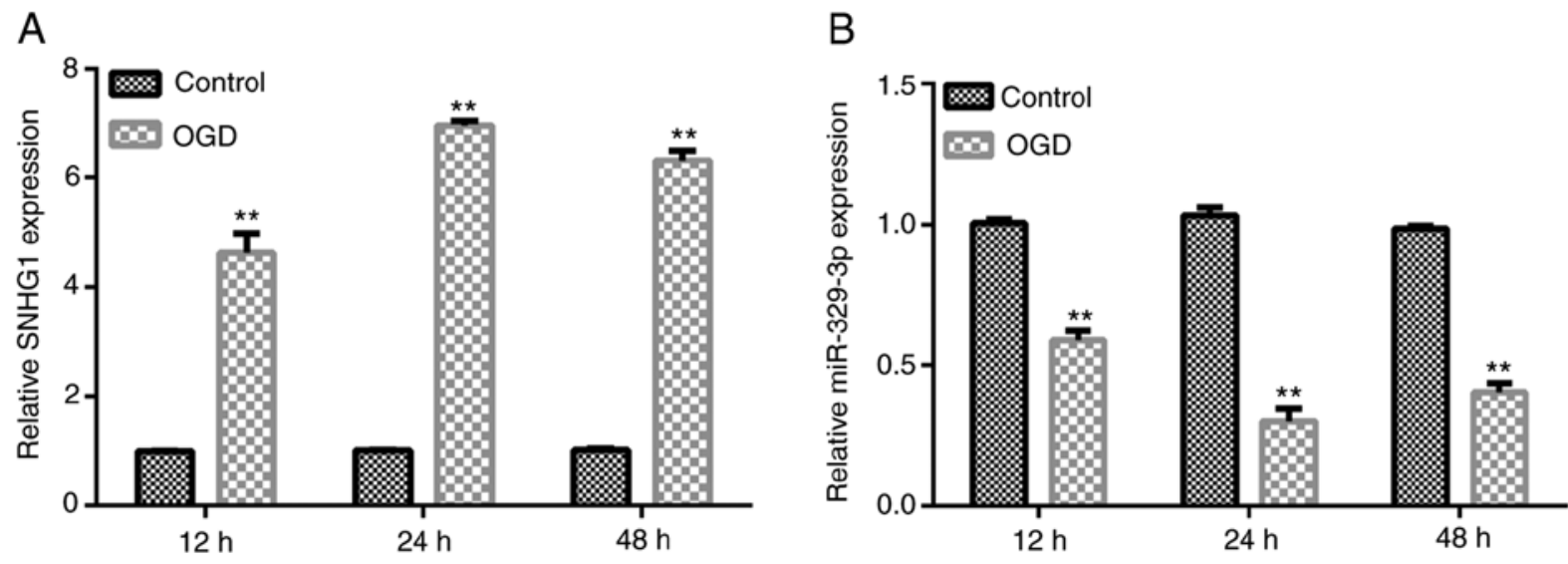

Figure 2. Expression levels of SNHG1 and miR-329-3p in OGD-induced BV-2 cells. BV-2 cells were cultured in serum/glucose-free DMEM with 95\% $\mathrm{N}_{2}$ and $5 \% \mathrm{CO}_{2}$ at $37^{\circ} \mathrm{C}$ for 12,24 or $48 \mathrm{~h}$. Then, relative mRNA expression levels of (A) SNHG1 and (B) miR-329-3p in BV-2 cells were analyzed using reverse transcription-quantitative PCR. ${ }^{* *} \mathrm{P}<0.01$ vs. control. SNHG1, small nucleolar RNA host gene 1; miR, microRNA; OGD, oxygen-glucose deprivation.
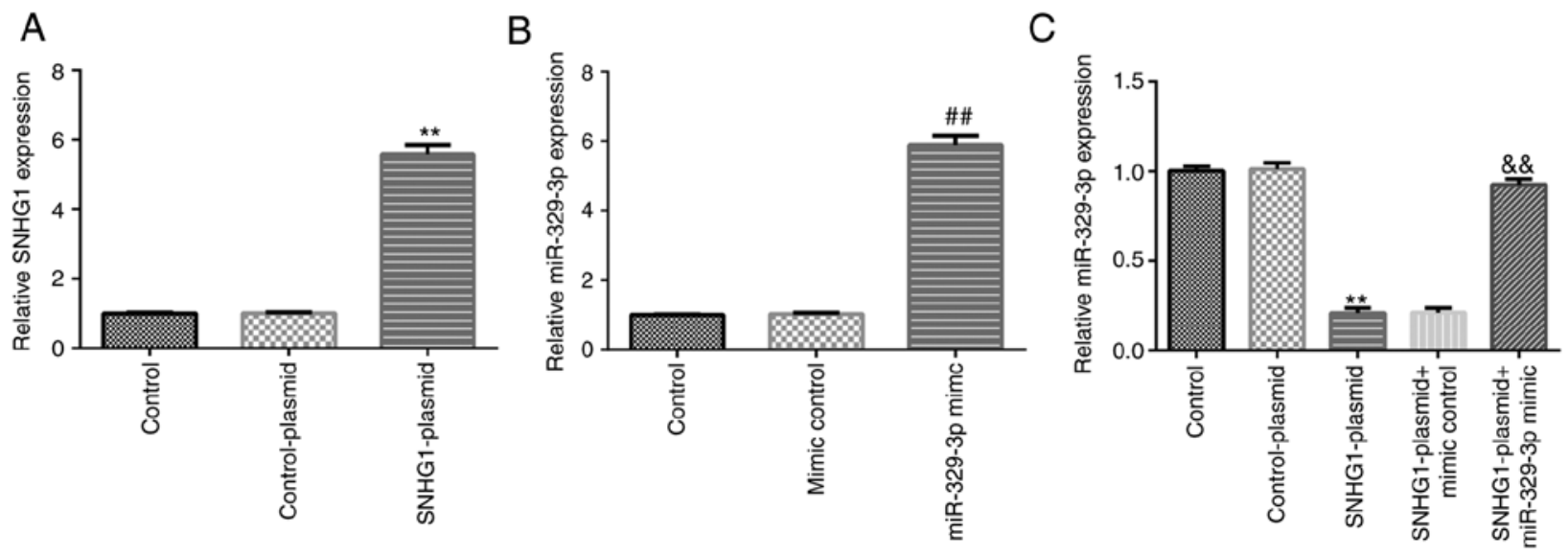

Figure 3. Transfection efficiency of SNHG1-plasmid and miR-329-3p mimic in BV-2 cells. BV-2 cells were transfected with control-plasmid, SNHG1-plasmid, mimic control, miR-329-3p mimic, SNHG1-plasmid + mimic control, or SNHG1-plasmid + miR-329-3p mimic for 24 h. (A) Reverse transcriptionquantitative PCR was performed to analyze the mRNA expression levels of SNHG1 in BV-2 cells following transfection with control-plasmid or SNHG1-plasmid. (B) Reverse transcription-quantitative PCR was performed to analyze the expression levels of miR-329-3p in BV-2 cells following transfection with mimic control or miR-329-3p mimic. (C) Reverse transcription-quantitative PCR was performed to analyze the expression levels of miR-329-3p in BV-2 cells transfected with control-plasmid, SNHG1-plasmid, SNHG1-plasmid + mimic control, or SNHG1-plasmid + miR-329-3p mimic. ${ }^{* *} \mathrm{P}<0.01$ vs. control-plasmid; ${ }^{\# \#} \mathrm{P}<0.01$ vs. mimic control; ${ }^{\& \&} \mathrm{P}<0.01$ vs. SNHG1-plasmid + mimic control. SNHG1, small nucleolar RNA host gene 1; miR, microRNA.

Expression levels of SNHG1 and miR-329-3p in the $O G D$-induced $B V-2$ cell model. An OGD cell model was established via OGD induction as previously described (25). After OGD induction for 12,24 or $48 \mathrm{~h}$, RT-qPCR was performed to analyze the expression levels of SNHG1 and miR-329-3p in BV-2 cells compared with the control group. The results revealed that the expression levels of SNHG1 were significantly upregulated, while the expression levels of miR-329-3p were significantly downregulated in the OGD group (Fig. 2A and B).

SNHG1-plasmid downregulates the expression levels of miR-329-3p in BV-2 cells. BV-2 cells were transfected with control-plasmid, SNHG1-plasmid, mimic control, miR-329-3p mimic, SNHG1-plasmid + mimic control or SNHG1-plasmid + miR-329-3p mimic, and the transfection efficiencies were determined using RT-qPCR. The results demonstrated that transfection with the SNHG1-plasmid or miR-329-3p mimic markedly upregulated the expression levels of SNHG1 and miR-329-3p, respectively, in BV-2 cells (Fig. 3A and B). Compared with the control-plasmid group, transfection with the SNHG1-plasmid significantly downregulated the expression levels of miR-329-3p in BV-2 cells, while this downregulation was reversed following transfection with the miR-329-3p mimic (Fig. 3C).

SNHG1 inhibits $O G D$-induced $B V-2$ cell activation by downregulating the expression of miR-329-3p. BV-2 cells were transfected for $24 \mathrm{~h}$ and then divided into the following six groups: i) Control group; ii) OGD group; iii) OGD + control-plasmid group; iv) OGD + SNHG1-plasmid group; v) OGD + SNHG1-plasmid + mimic control group; and vi) OGD + SNHG1-plasmid + miR-329-3p mimic group. Compared with the control group, the expression levels of SNHG1 were significantly upregulated (Fig. 4A), the expression levels of miR-329-3p were significantly downregulated (Fig. 4B), and the release of TNF- $\alpha$ and NO was significantly increased (Fig. 4C and D) in the OGD group. In addition, the 
A

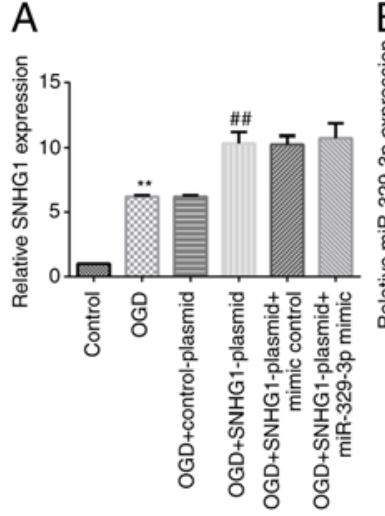

B $\quad C$

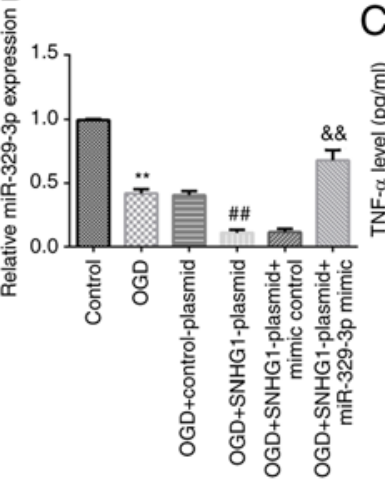

E
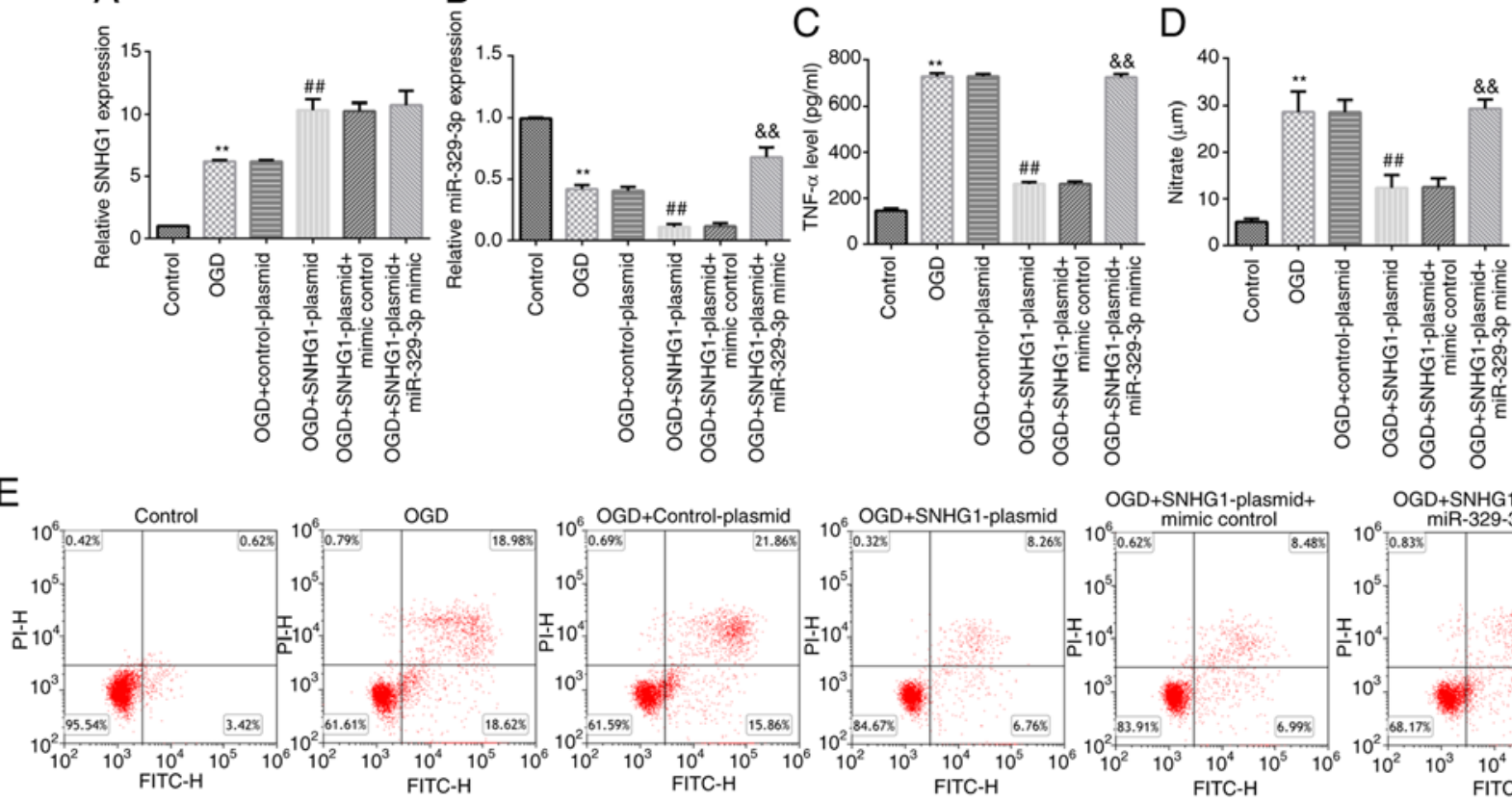

OGD+SNHG1-plasmid+

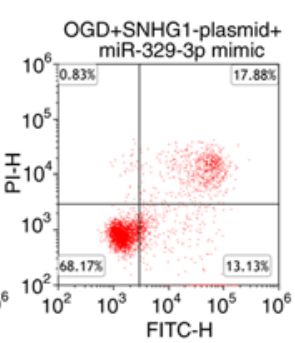

$\mathrm{F}$

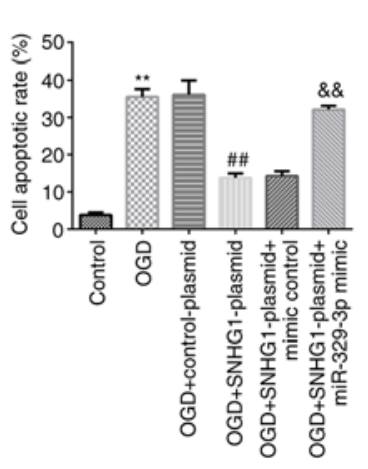

G

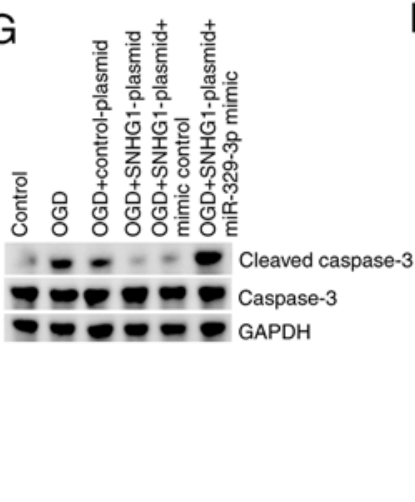

$\mathrm{H}$

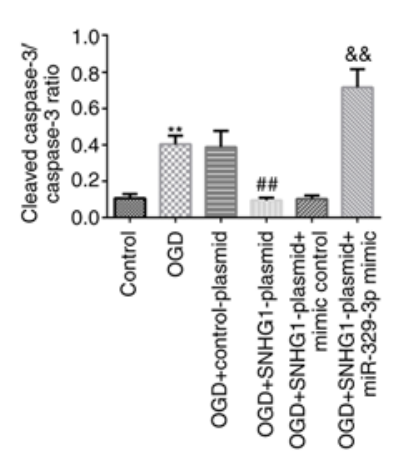

I

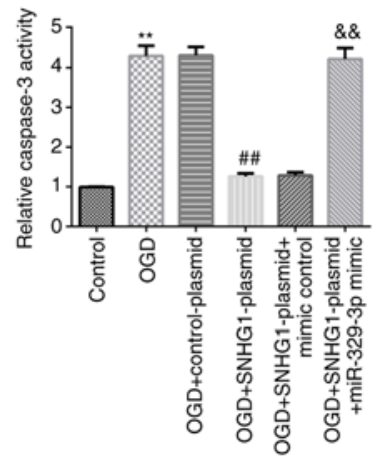

Figure 4. Overexpression of SNHG1 affects OGD-induced BV-2 cell activation and apoptosis. BV-2 cells were cultured in serum/glucose-free DMEM with $95 \% \mathrm{~N}_{2}$ and $5 \% \mathrm{CO}_{2}$ at $37^{\circ} \mathrm{C}$ for $48 \mathrm{~h}$, then the cells were transfected with control-plasmid, SNHG1-plasmid, SNHG1-plasmid + mimic control, or SNHG1-plasmid + miR-329-3p mimic for another $24 \mathrm{~h}$. Reverse transcription-quantitative PCR was performed to analyze the expression levels of (A) SNHG1 and (B) miR-329-3p in BV-2 cells. (C) TNF- $\alpha$ release into the supernatant of the transfected cells was detected using an ELISA. (D) NO production in the supernatant of the transfected cells was detected using a commercial kit. (E and F) Flow cytometric analysis was used to measure the levels of apoptosis of BV-2 cells induced by OGD. (G) Cleaved caspase-3 and caspase-3 protein expression was analyzed using western blotting. (H) Cleaved caspase-3/caspase-3 ratio. (I) Caspase-3 activity was measured using a colorimetric assay kit. ${ }^{* *} \mathrm{P}<0.01$ vs. control; ${ }^{\# \#} \mathrm{P}<0.01$ vs. OGD + control-plasmid; \&\& $\mathrm{P}<0.01 \mathrm{vs}$. OGD + SNH G1-plasmid + mimic control. SNHG1, small nucleolar RNA host gene 1; miR, microRNA; OGD, oxygen-glucose deprivation; NO, nitric oxide.

results of the flow cytometry, caspase-3 activity and western blot analyses demonstrated that the levels of cell apoptosis, caspase- 3 activity, cleaved caspase- 3 protein expression and the cleaved caspase-3/caspase-3 ratio were all markedly increased in the OGD group compared with the control group (Fig. 4E-I).

Similarly, compared with the OGD + control-plasmid group, SNHG1 expression levels were upregulated in the OGD + SNHG1-plasmid group, while miR-329-3p expression levels, TNF- $\alpha$ and NO release, cell apoptosis, cleaved caspase- 3 protein expression, the cleaved caspase-3/caspase- 3 ratio and caspase-3 activity were all significantly reduced in the OGD + SNHG1-plasmid group. All these effects were significantly reversed following transfection with the miR-392-3p mimic.

Knockdown of miR-329-3p expression inhibits OGD-induced $B V-2$ cell activation. The transfection efficiency of the
miR-329-3p inhibitor into BV-2 cells was detected $24 \mathrm{~h}$ post-transfection, and the results revealed that the miR-329-3p inhibitor significantly downregulated the expression levels of miR-329-3p in BV-2 cells (Fig. 5A). The cells were then divided into the following four groups: i) Control group; ii) OGD group; iii) OGD + inhibitor control; and iv) OGD + miR-329-3p inhibitor group. Experiments were subsequently performed to determine the molecular mechanisms underlying the effects of miR-329-3p on BV-2 cells. As shown in Fig. 5B-I, compared with the control group, miR-329-3p expression levels were downregulated in the OGD group, while TNF- $\alpha$ and NO release, cell apoptosis, cleaved caspase- 3 protein expression, the cleaved caspase-3/caspase- 3 ratio and caspase- 3 activity were all significantly increased in the OGD group. Similarly, compared with the OGD + inhibitor control group, miR-329-3p expression, TNF- $\alpha$ and NO release, cell apoptosis, cleaved caspase-3 protein expression, the cleaved caspase-3/caspase-3 

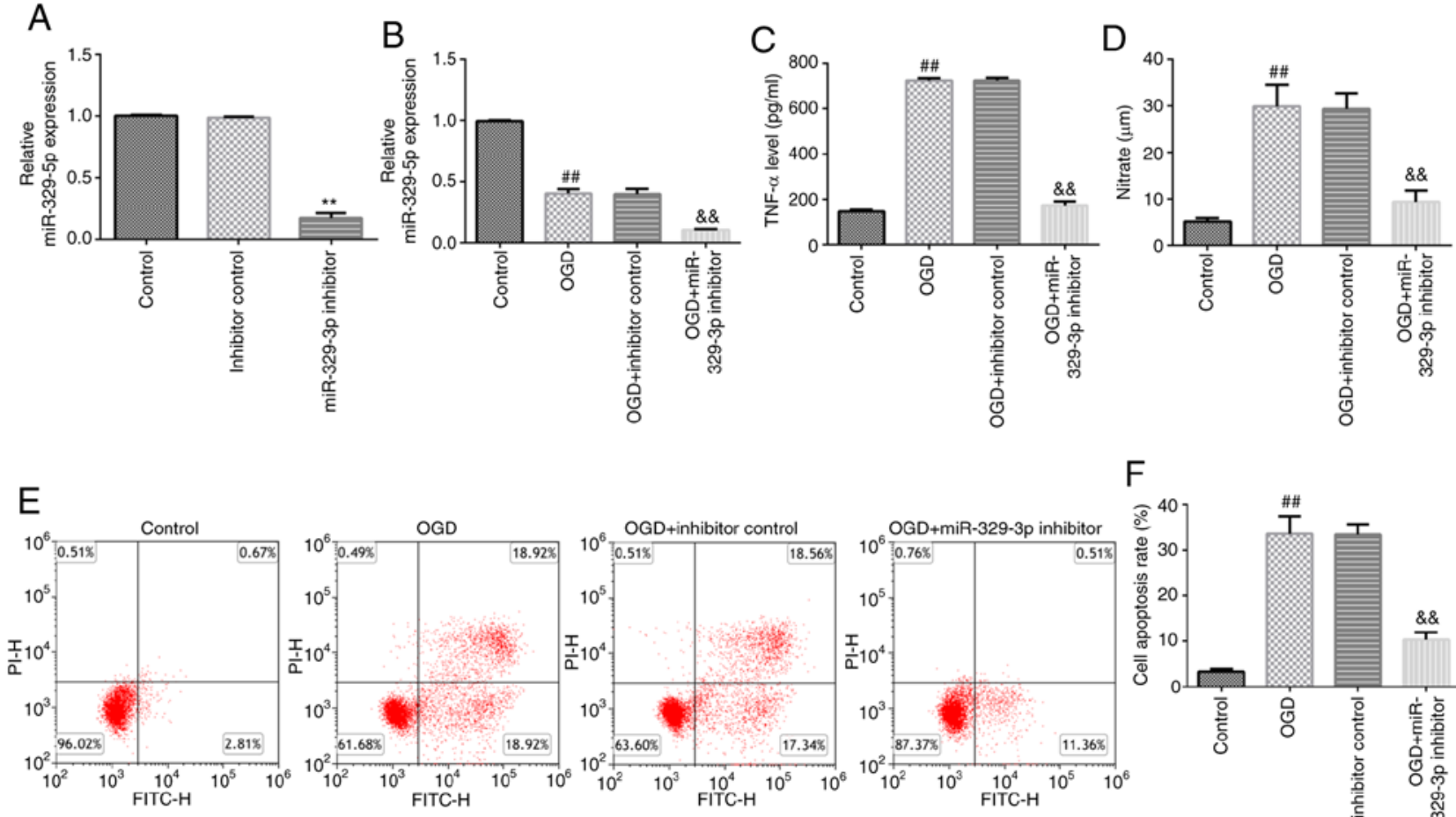

$\mathrm{F}$
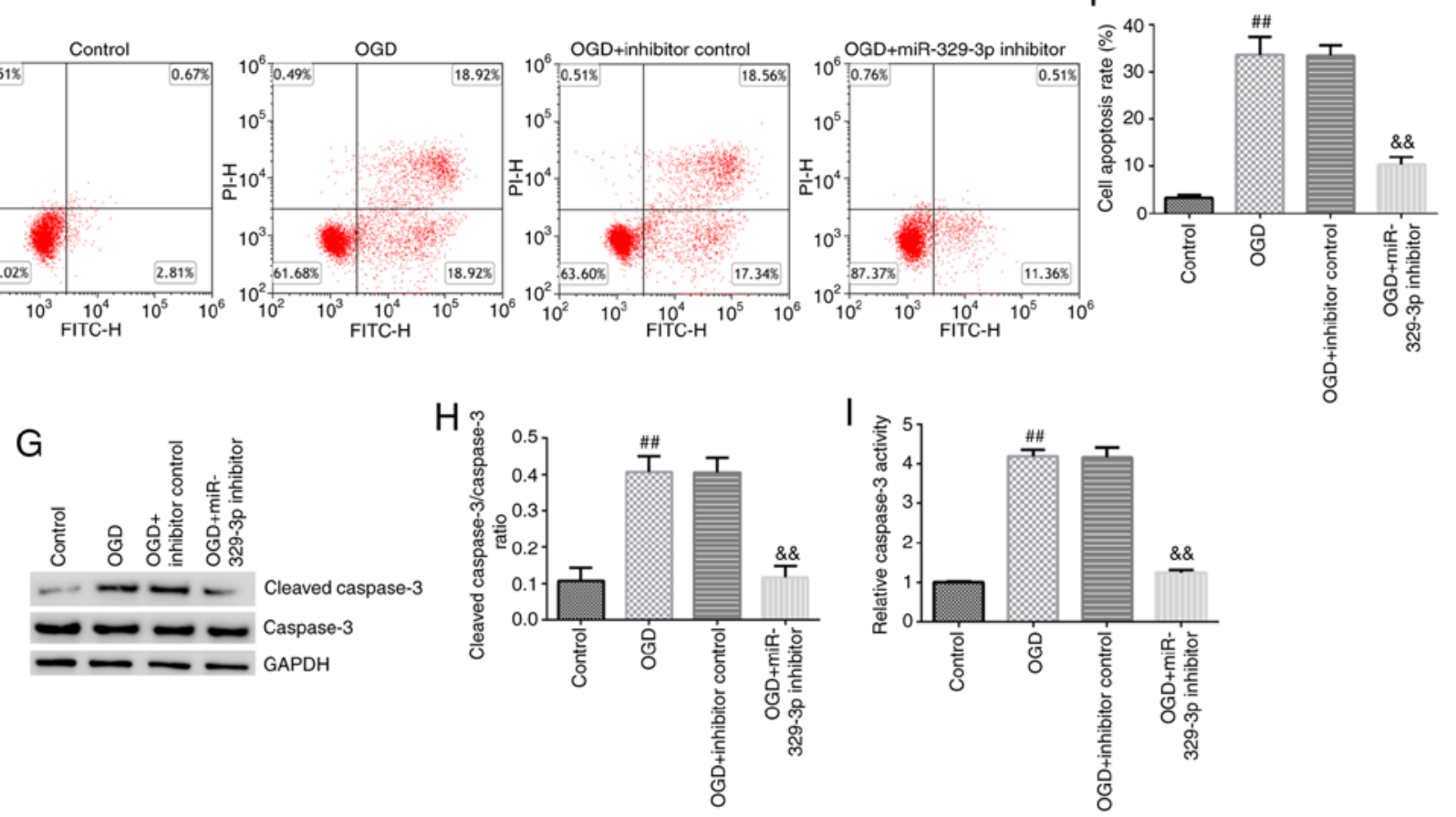

Figure 5. Knockdown of miR-329-3p expression affects OGD-induced BV-2 cell activation and apoptosis. (A) BV-2 cells were transfected with inhibitor control or miR-329-3p inhibitor for $24 \mathrm{~h}$, and then reverse transcription-quantitative PCR was performed to analyze the expression levels of miR-329-3p. (B) BV-2 cells were cultured in serum/glucose-free DMEM with $95 \% \mathrm{~N}_{2}$ and $5 \% \mathrm{CO}_{2}$ at $37^{\circ} \mathrm{C}$ for $48 \mathrm{~h}$; then, the cells were transfected with inhibitor control or miR-329-3p inhibitor for $24 \mathrm{~h}$ and reverse transcription-quantitative PCR was performed to analyze the expression levels of miR-329-3p. (C) TNF- $\alpha$ release into the supernatant of the transfected cells was detected using ELISA. (D) NO production in the supernatant of the transfected cells was detected using a commercial kit. (E and F) Flow cytometric analysis was used to measure the levels of apoptosis of BV-2 cells induced by OGD. (G) Cleaved caspase-3 and caspase-3 protein expression was measured using western blotting. (H) Cleaved caspase-3/caspase-3 ratio. (I) Caspase-3 activity was detected using a colorimetric assay kit. ${ }^{* *} \mathrm{P}<0.01$ vs. inhibitor control; ${ }^{\# \#} \mathrm{P}<0.01$ vs. control; ${ }^{\&}{ }^{\&} \mathrm{P}<0.01$ vs. OGD + inhibitor control. SNHG1, small nucleolar RNA host gene 1; miR, microRNA; OGD, oxygen-glucose deprivation; $\mathrm{NO}$, nitric oxide.

ratio and caspase- 3 activity were all markedly decreased in the OGD + miR-329-3p inhibitor group.

\section{Discussion}

Cerebral infarction is caused by blood circulation disorders, which lead to ischemia, hypoxia and necrosis of the brain areas affected (1-3). The clinical symptoms of cerebral infarction are complex, and are associated with the location of the brain damage, the size of the cerebral ischemic blood vessels, the severity of the ischemia, the influence of other diseases prior to the onset of ischemia and the effects of the comorbidity of cerebral infarction and other types of vital organ disease. Mild cases of cerebral infarction can be completely asymptomatic, but they can also manifest as recurrent limb paralysis or dizziness, which is known as a transient ischemic attack. Individuals with severe cases of cerebral infarction may not only experience limb paralysis, but acute coma and death may also occur in some cases $(28,29)$.

The expression levels of SNHG1 were previously found to be upregulated in middle cerebral artery occlusion model mice (30), indicating the potentially important role of SNHG1 in cerebral infarction. Furthermore, the level of SNHG1 is elevated in OGD and cerebral ischemic rodents and exerts neuroprotective effects involving the PI3K/AKT pathway (20). To investigate the mechanisms underlying the role of lncRNA SNHG1 in cerebral infarction, the present study predicted and verified the binding site between miR-329-3p and SNHG1. Microglia are small cells found in the nervous system, which are mostly localized in the gray matter near the cell bodies of 
neurons and around small blood vessels, but can also be found in the white matter of the brain (31). Microglia can promote the development of the nervous system and regulate the number of neurons in the central nervous system (32). After an ischemic stroke, activated microglia were discovered to promote the excessive release of inflammatory cytokines, thereby exacerbating neuronal damage $(33,34)$. Therefore, regulating the activation of endogenous microglia may represent a new treatment target in ischemic stroke $(22,23)$. Furthermore, OGD induced BV-2 cells have been widely used to study cerebral infarction in vitro (24-26). In the present study, BV-2 cells were used to establish an in vitro OGD model, and the results revealed that the expression levels of SNHG1 were upregulated in OGD-induced cells, while miR-329-3p expression levels were downregulated.

To determine whether SNHG1 affected the activation of BV-2 cells by regulating miR-329-3p expression, experiments with BV-2 cells were performed following overexpression of SNHG1 and downregulation of miR-329-3p. The results demonstrated that transfection with the SNHG1-plasmid downregulated miR-329-3p expression, while this effect was reversed following transfection with the miR-329-3p mimic. TNF- $\alpha$ and NO are important proinflammatory factors that have been found to play important roles in the occurrence and development of the systemic inflammatory response $(35,36)$. Thus, the present study measured the release of TNF- $\alpha$ and NO in the supernatant of OGD-induced BV-2 cells. The results revealed that the overexpression of SNHG1 or knockdown of miR-329-3p expression inhibited the release of TNF- $\alpha$ and NO in the supernatant of OGD-induced BV-2 cells. Contrary to our findings, an in vitro study on Parkinson's disease demonstrated that LPS increased SNHG1 expression in BV-2 cells, leading to NLRP3 activation and cytokine production (37). Moreover, the findings of present study indicated that the overexpression of SNHG1 or knockdown of miR-329-3p expression reduced OGD-induced BV-2 cell apoptosis. Of note, all the effects of SNHG1 overexpression on OGD-induced BV-2 cells were significantly reversed by miR-329-3p overexpression. Other previous studies have also reported that SNHG1 may act as a sponge RNA to interfere with the actions of target miRNAs $(38,39)$. The findings of the present study confirmed that the effects of SNHG1 on OGD-induced BV-2 cells are mediated through downregulating miR-329-3p levels.

The present study was only a preliminary in vitro study on the role of IncRNA SNHG1 in cerebral infarction. In order to further elucidate the role of lncRNA SNHG1 in cerebral infarction, extensive in-depth research is required. For example, the role of lncRNA SNHG1 silencing or miR-329-3p overexpression alone in cerebral infarction should be investigated. Furthermore, the downstream targets of SNHG1 and miR-329-3p should be further analyzed. In addition, the role of lncRNA SNHG1 and miR-329-3p in cerebral infarction should be studied in vivo. These issues will be addressed in future studies.

In conclusion, the results of the present study indicated that lncRNA SNHG1 may play an important role in microglia activation by modulating the expression of miR-329-3p. Therefore, targeting SNHG1 and miR-329-3p may provide novel strategies for the treatment of cerebral infarction.

\section{Acknowledgements}

Not applicable.

\section{Funding}

The present study was supported by the Science and Technology Development Foundation of Nanjing Medical University (grant no. 2017NJMU083), the Zhejiang Chinese Medical University Field of Scientific Research Foundation (grant no. 2018ZY23) and the Hangzhou Agriculture and Social Development Research Project Foundation (grant no. 20191203B84).

\section{Availability of data and materials}

The datasets used and/or analyzed during the current study are available from the corresponding author on reasonable request.

\section{Authors' contributions}

JH designed the study, in addition to performing all experiments, analyzing the data and preparing the manuscript. XX, MJ, JL, and NL contributed to performing the experiments and data collection. TN analyzed and interpreted the data, and revised the manuscript. JH and TN confirm the authenticity of all the raw data. All the authors have read and approved the final manuscript.

\section{Ethics approval and consent to participate}

Not applicable.

\section{Patient consent for publication}

Not applicable.

\section{Competing interests}

The authors declare that they have no competing interests.

\section{References}

1. Sun W, Li G, Zeng X, Lai Z, Wang M, Ouyang Y, Zeng G, Peng J, Zhong J, Xiao D, et al: Clinical and imaging characteristics of cerebral infarction in patients with nonvalvular atrial fibrillation combined with cerebral artery stenosis. J Atheroscler Thromb 25: 720-732, 2018.

2. Choi JW, Chong S, Phi JH, Lee JY, Kim HS, Chae JH, Lee J and Kim SK: Postoperative symptomatic cerebral infarction in pediatric moyamoya disease: Risk factors and clinical outcome. World Neurosurg 136: e158-e164, 2020.

3. Wang YW and Zhang GM: New silent cerebral infarction in patients with acute non-cerebral amyloid angiopathy intracerebral hemorrhage as a predictor of recurrent cerebrovascular events. Med Sci Monit 25: 418-426, 2019.

4. Sveinsson OA, Kjartansson O and Valdimarsson EM: Cerebral ischemia/infarction-epidemiology, causes and symptoms. Laeknabladid 100: 271-279, 2014 (In Icelandic).

5. Rojsanga W, Sawanyawisuth K, Chotmongkol V, Tiamkao S, Kongbonkiat $\mathrm{K}$ and Kasemsap N: Clinical risk factors predictive of thrombotic stroke with large cerebral infarction. Neurol Int 11: 7941, 2019

6. Jin L, Zhou J, Shi W, Xu L, Sheng J, Fan J, Yuan Y and Yuan H: Effects of six types of aspirin combination medications for treatment of acute cerebral infarction in China: A network meta-analysis. J Clin Pharm Ther 44: 91-101, 2019. 
7. Orlický M, Hrbáč T, Sameš M, Vachata P, Hejčl A, Otáhal D, Havelka J, Netuka D, Herzig R, Langová K and Śkoloudík D: Anesthesia type determines risk of cerebral infarction after carotid endarterectomy. J Vasc Surg 70: 138-147, 2019.

8. Tu J, Wang LX, Wen HF, Xu YC and Wang PF: The association of different types of cerebral infarction with post-stroke depression and cognitive impairment. Medicine (Baltimore) 97: e10919, 2018.

9. Xu W, Xie N, Zhang C and Huang Q: Imaging characteristics and pathogenesis of intracranial artery stenosis in patients with acute cerebral infarction. Exp Ther Med 15: 4564-4570, 2018.

10. Rodríguez-Gómez JA, Kavanagh E, Engskog-Vlachos P, Engskog MKR, Herrera AJ, Espinosa-Oliva AM, Joseph B, Hajji N, Venero JL and Burguillos MA: Microglia: Agents of the CNS pro-inflammatory response. Cells 9: 1717, 2020.

11. Kinuthia UM, Wolf A and Langmann T: Microglia and inflammatory responses in diabetic retinopathy. Front Immunol 11: 564077, 2020.

12. Szcześniak MW, Wanowska E, Mukherjee N, Ohler U and Makałowska I: Towards a deeper annotation of human lncRNAs. Biochim Biophys Acta Gene Regul Mech 1863: 194385, 2020.

13. Puvvula PK: LncRNAs regulatory networks in cellular senescence. Int J Mol Sci 20: 2615, 2019.

14. Simion V, Haemmig S and Feinberg MW: LncRNAs in vascular biology and disease. Vascul Pharmacol 114: 145-156, 2019.

15. Lekka E and Hall J: Noncoding RNAs in disease. FEBS Lett 592 2884-2900, 2018.

16. Dinescu S, Ignat S, Lazar AD, Constantin C, Neagu $M$ and Costache M: Epitranscriptomic signatures in lncRNAs and their possible roles in cancer. Genes (Basel) 10: 52, 2019.

17. Shi YL, Wang Q and Wei JC: Influence of lncRNA-MALAT1 on neuronal apoptosis in rats with cerebral infarction through regulating the ERK/MAPK signaling pathway. Eur Rev Med Pharmacol Sci 23: 8039-8048, 2019.

18. Shen J, Zhao Z, Shang W, Liu C, Zhang B, Xu Z and Cai H Fabrication of a nano polymer wrapping Meg3 ShRNA plasmid for the treatment of cerebral infarction. Artif Cells Nanomed Biotechnol 46 (Suppl 2): S894-S903, 2018.

19. Zhao JH, Wang B, Wang XH, Wang JR and Xu CW: Influence of lncRNA ANRIL on neuronal apoptosis in rats with cerebral infarction by regulating the NF- $\kappa \mathrm{B}$ signaling pathway. Eur Rev Med Pharmacol Sci 23: 10092-10100, 2019.

20. Chen J, Zhang W, Wu YQ, Chen $\mathrm{H}$ and Zhao JF: LncRNA SNHG1 inhibits neuronal apoptosis in cerebral infarction rats through PI3K/Akt signaling pathway. Eur Rev Med Pharmaco Sci 23: 5366-5373, 2019.

21. Huang L, Jiang X, Wang Z, Zhong X, Tai S and Cui Y: Small nucleolar RNA host gene 1: A new biomarker and therapeutic target for cancers. Pathol Res Pract 214: 1247-1252, 2018.

22. Kaur C, Sivakumar V, Zou Z and Ling EA: Microglia-derived proinflammatory cytokines tumor necrosis factor-alpha and interleukin-lbeta induce Purkinje neuronal apoptosis via their receptors in hypoxic neonatal rat brain. Brain Struct Funct 219 151-170, 2014.

23. Ma Y, Wang J, Wang Y and Yang GY: The biphasic function of microglia in ischemic stroke. Prog Neurobiol 157: 247-272, 2017.
24. Mo Z, Tang C, Li H, Lei J, Zhu L, Kou L, Li H, Luo S, Li C, Chen $\mathrm{W}$ and Zhang L: Eicosapentaenoic acid prevents inflammation induced by acute cerebral infarction through inhibition of NLRP3 inflammasome activation. Life Sci 242: 117133, 2020.

25. Qi X, Shao M, Sun H, Shen Y, Meng D and Huo W: Long non-coding RNA SNHG14 promotes microglia activation by regulating miR-145-5p/PLA2G4A in cerebral infarction. Neuroscience 348: 98-106, 2017.

26. Lu D, Shen L, Mai H, Zang J, Liu Y, Tsang CK, Li K and Xu A: HMG-CoA reductase inhibitors attenuate neuronal damage by suppressing oxygen glucose deprivation-induced activated microglial cells. Neural Plast 2019: 7675496, 2019.

27. Livak KJ and Schmittgen TD: Analysis of relative gene expression data using real-time quantitative PCR and the 2(-Delta Delta C(T)) method. Methods 25: 402-408, 2001.

28. Chen XY, Wang Q, Wang X and Wong KS: Clinical features of thalamic stroke. Curr Treat Options Neurol 19: 5, 2017.

29. Liang Y, Wu J, Liu J, Liu H and Chen J: The clinical implications of thrombelastography in the diagnosis of acute cerebral infarction. Clin Lab 64: 147-152, 2018.

30. Zhang L, Luo X, Chen F, Yuan W, Xiao X, Zhang X, Dong Y, Zhang Y and Liu Y: LncRNA SNHG1 regulates cerebrovascular pathologies as a competing endogenous RNA through HIF-1 $\alpha$ /VEGF signaling in ischemic stroke. J Cell Biochem 119: 5460-5472, 2018.

31. Prinz M, Jung S and Priller J: Microglia biology: One century of evolving concepts. Cell 179: 292-311, 2019.

32. Nayak D, Roth TL and McGavern DB: Microglia development and function. Annu Rev Immunol 32: 367-402, 2014.

33. Wolf SA, Boddeke HW and Kettenmann H: Microglia in physiology and disease. Annu Rev Physiol 79: 619-643, 2017.

34. Bartels T, De Schepper S and Hong S: Microglia modulate neurodegeneration in Alzheimer's and Parkinson's diseases. Science 370: 66-69, 2020

35. Aggarwal R, Jain AK, Mittal P, Kohli M, Jawanjal $P$ and Rath G: Association of pro- and anti-inflammatory cytokines in preeclampsia. J Clin Lab Anal 33: e22834, 2019.

36. Li Q, Hu X, Xuan Y, Ying J, Fei Y, Rong J, Zhang Y, Zhang J, Liu C and Liu Z: Kaempferol protects ethanol-induced gastric ulcers in mice via pro-inflammatory cytokines and NO. Acta Biochim Biophys Sin (Shanghai) 50: 246-253, 2018.

37. Cao B, Wang T, Qu Q, Kang T and Yang Q: Long noncoding RNA SNHG1 promotes neuroinflammation in Parkinson's disease via regulating miR-7/NLRP3 pathway. Neuroscience 388: 118-127, 2018.

38. Deng R, Zhang J and Chen J: lncRNA SNHG1 negatively regulates miRNA-101-3p to enhance the expression of ROCK1 and promote cell proliferation, migration and invasion in osteosarcoma. Int J Mol Med 43: 1157-1166, 2019.

39. Yan SM, Li H, Shu Q, Wu WJ, Luo XM and Lu L: LncRNA SNHG1 exerts a protective role in cardiomyocytes hypertrophy via targeting miR-15a-5p/HMGA1 axis. Cell Biol Int 44: 1009-1019, 2020 .

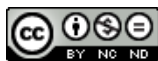

This work is licensed under a Creative Commons

Attribution-NonCommercial-NoDerivatives 4.0 International (CC BY-NC-ND 4.0) License. 